9-2003

\title{
A Novel Method for Measuring Induction Machine Magnetizing Inductance
}

Ana Vladan Stankovic

Cleveland State University, a.stankovic@csuohio.edu

Eric L. Benedict

University of Wisconsin - Madison, benedict@cae.wisc.edu

Vinod John

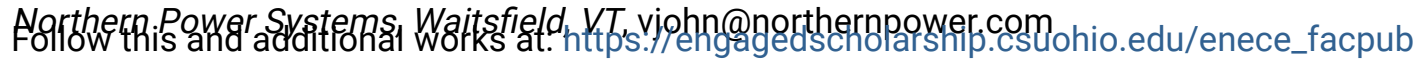

qParsoftehlepectromagnetics and Photonics Commons

Hniversity of Wisconsin Madis@n lipo@engr.wiscedu know!

\section{Publisher's Statement}

(C) 2003 IEEE. Personal use of this material is permitted. Permission from IEEE must be obtained for all other users, including reprinting/ republishing this material for advertising or promotional purposes, creating new collective works for resale or redistribution to servers or lists, or reuse of any copyrighted components of this work in other works.

\section{Original Citation}

Stankovic, A. V., Benedict, E. L., John, V., \& Lipo, T. A. (2003). A novel method for measuring induction machine magnetizing inductance. IEEE Transactions on Industry Applications, 39, 5, 1257-1263.

\section{Repository Citation}

Stankovic, Ana Vladan; Benedict, Eric L.; John, Vinod; and Lipo, Thomas A., "A Novel Method for Measuring Induction Machine Magnetizing Inductance" (2003). Electrical Engineering \& Computer Science Faculty Publications. 109.

https://engagedscholarship.csuohio.edu/enece_facpub/109

This Article is brought to you for free and open access by the Electrical Engineering \& Computer Science Department at EngagedScholarship@CSU. It has been accepted for inclusion in Electrical Engineering \& Computer Science Faculty Publications by an authorized administrator of EngagedScholarship@CSU. For more information, please contact library.es@csuohio.edu. 


\title{
A Novel Method for Measuring Induction Machine Magnetizing Inductance
}

\author{
Ana Vladan Stanković, Member, IEEE, Eric L. Benedict, Student Member, IEEE, Vinod John, Member, IEEE, and
} Thomas A. Lipo, Fellow, IEEE

\begin{abstract}
This paper presents a novel method for measuring the magnetizing inductance of an induction machine. The approach uses a static de excitation technique which can be employed whenever the neutral of the machine is accessible. The proposed method measures only the magnetizing inductance and not the self inductance which normally includes the effect of the stator leakage inductance. Because this test uses a dc excitation, the iron losses in the motor are considerably reduced as well and minimally influence the measurement when compared to the traditional $60-\mathrm{Hz}$ no-load test. By using the proposed method for measuring only the magnetizing inductance, the stator leakage inductance can be later individually determined by performing a separate no-load test. Test results using the method are compared with theoretical values and confirm its feasibility.
\end{abstract}

Index Terms-DC test, inductance measurement, induction machines, leakage inductance, locked-rotor test, magnetizing inductance, no-load test.

\section{INTRODUCTION}

$\mathbf{T}$ RADITIONALLY, the magnetizing inductance along with other induction machine parameters have been measured by performing $60-\mathrm{Hz}$ no-load and locked-rotor tests [1]-[4]. The values of the rated voltage and the no-load current at rated voltage and frequency are used to calculate the magnetizing inductance under the assumption that the leakage (measured by locked-rotor test) has been split into arbitrary stator and rotor portions (typically half for each). In order to improve the estimate of the stator and rotor leakage inductances, an iterative procedure must be applied to account for the effect of the magnetizing inductance. More recently, static tests using time domain [5], [6] and frequency-domain [7] techniques have been proposed for measuring the induction machine parameters which require heavy computational overhead.

This paper proposes a simple, static (nonrotating) method for measuring the magnetizing inductance of an induction machine

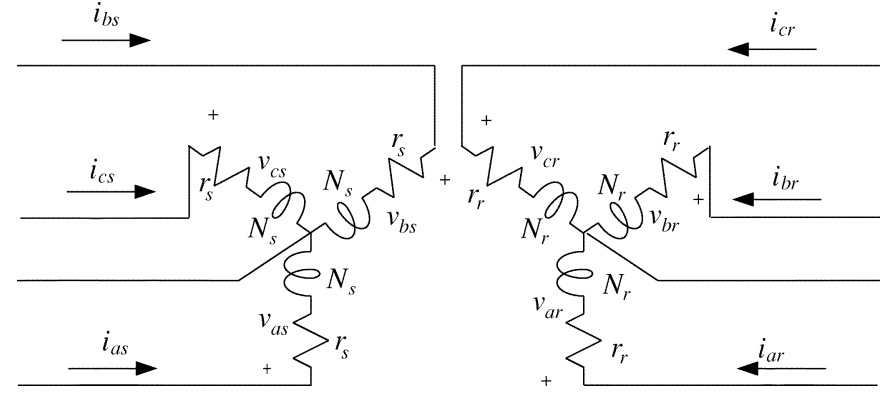

Fig. 1. Induction machine circuit model.

using only a switched dc excitation. An accurate value for the magnetizing inductance is obtained with the proposed method because it directly measures only the magnetizing portion of the stator self inductance whereas the traditional no-load test measures the total self inductance which includes the effects of leakage flux. Since the test essentially a dc measurement, the influence of the iron loss is also reduced when the proposed method is applied.

The proposed method follows the pioneering work of C. V. Jones [8]. His work, proposed in 1967, involves the direct current measurement of rotational self inductances. However, unlike the proposed test, the method described by Jones does not utilize the open circuit voltage of an adjoining phase to determine the magnetizing inductance which is a much easier measurement than the one proposed by Jones.

With the introduction of vector controlled drives, the ability to measure all of the machine parameters in situ is important for applications where adaptive models have been implemented to estimate flux position, rotor speed, etc. The proposed test can be used to obtain the magnetizing inductance without rotating the machine. When combined with the locked-rotor test, the complete machine model can be determined by assuming that the leakage inductance is split in a prescribed manner between the rotor and stator. Alternatively, if a nonestimated leakage inductance is desired, the proposed test can be combined with a no-load test and the primary leakage inductance can be directly determined.

\section{TheOretical Derivation}

The induction machine can be represented by the circuit model shown in Fig. 1. If the rotor is assumed to have a squirrel cage construction, then the effective rotor phase windings can be assumed to be spatially aligned with the stator windings. 


\section{A. Flux Linkages} [9]

The flux linkage equation for the stator $a$-phase winding is

$$
\begin{aligned}
\lambda_{a s}=\left(L_{l s}+L_{m s}\right) & i_{a s}-\frac{L_{m s}}{2} i_{b s} \\
& -\frac{L_{m s}}{2} i_{c s}+L_{s r} i_{a r}-\frac{L_{s r}}{2} i_{b r}-\frac{L_{s r}}{2} i_{c r}
\end{aligned}
$$

where $i_{a s}, i_{b s}$, and $i_{c s}$ are the stator currents and $i_{a r}, i_{b r}$, and $i_{c r}$ are the rotor currents referred to the stationary reference frame. The inductance $L_{l s}$ represents the equivalent stator leakage, $L_{m s}$ is the fundamental stator mutual inductance, and $L_{s r}$ is the stator-rotor mutual inductance.

Note that the $a$-phase leakage flux, $L_{l s} i_{a s}$, is a simplification of several different flux components which are combined to form the equivalent leakage inductance under normal operation. These leakage fluxes include the slot leakage and end-winding fluxes. In addition to these traditional leakage fluxes, the physical construction of the stator windings introduces space harmonic flux components which do not contribute substantially to the average torque. Consequently, these flux components are often considered to produce the harmonic or differential leakage flux which consists of the zigzag and belt leakage components [10].

Since the operating conditions of the proposed test are different from normal operation, it is important to examine these flux components under the proposed operating conditions. Because of the nature of the proposed excitation, the slot leakage, end-winding, and the space harmonic flux components need to be considered explicitly. The slot leakage and the end-winding inductances arise from similar mechanisms and can be considered at the same time. The differential leakage inductance will be examined subsequently.

The slot leakage flux linkage can be written as [11]

$$
\lambda_{s l a}=L_{s l s} i_{a s}-L_{s l m} i_{b s}-L_{s l m} i_{c s}
$$

where $L_{s l s}$ represents the slot self-inductance, and $L_{s l m}$ represents the slot mutual inductance. The end-winding inductance has a similar form

$$
\lambda_{\text {ewa }}=L_{\text {ews }} i_{\text {as }}-L_{\text {ewm }} i_{b s}-L_{\text {ewm }} i_{c s}
$$

where $L_{\text {ews }}$ represents the end-winding self-inductance and $L_{\text {ewm }}$ represents the end-winding mutual inductance.

Because the windings are not sinusoidally distributed, space harmonics are present and this should be accounted for. The harmonic winding factor $k_{h}$ is defined to be

$$
k_{h}=k_{p h} \cdot k_{d h}=\sin \left(h \frac{W}{\tau_{p}} \frac{\pi}{2}\right) \cdot \frac{\sin \left(h q \frac{\gamma}{2}\right)}{q \sin \left(h \frac{\gamma}{2}\right)}
$$

where $h$ is the space harmonic number, $W / \tau_{p}$ is the winding pitch, $q$ is the number of slots per phase belt, and $\gamma$ is the electrical angle between two slots [11]. This can be used to express the MMF, $\mathcal{F}_{p}$, generated by a singly excited phase

$$
\mathcal{F}_{p}(\theta)=\frac{4}{\pi} \frac{N_{s}}{P} I_{m} \sum_{h \text { odd }} \frac{k_{h}}{h} \cos \left(\frac{P h \theta}{2}\right)
$$

where $N_{s}$ is the equivalent number of series connected turns, $P$ is the number of poles, $I_{m}$ is the magnitude of the phase current, and $\theta$ is the mechanical angle between the position on the air gap and the center of the south pole. When the machine is operated under balanced three-phase excitation, it can be shown that the resulting magnetomotive force (MMF) $\mathcal{F}_{3 \Phi}$ is

$$
\mathcal{F}_{3 \Phi}=\frac{3}{2} \mathcal{F}_{p} .
$$

Also note that half of the flux generated by one phase couples each of the other two stator phases because of the $120^{\circ}$ spacing of the windings. The flux linkages coupling the $P$ poles of the $h^{\text {th }}$ MMF harmonic are

$$
\lambda_{h}=N_{s} \frac{k_{h}}{h} \frac{2}{\pi} \frac{\mu_{0}}{g_{e}^{\prime}} \tau_{p} l_{e} \mathcal{F}_{p h}
$$

where $\mu_{0}$ is the permeability of free space, $g_{e}^{\prime}$ is the effective air gap, $\tau_{p}$ is the pole length, and $l_{e}$ is the machine's stack length.

Thus, the harmonic mutual inductance between two phases can be found to be

$$
L_{m h, h}(h)=\frac{\lambda_{h}}{I_{m}}=\frac{N_{s}^{2}}{P} \frac{k_{h}^{2}}{h^{2}} \frac{4}{\pi^{2}} \mu_{0} \frac{\tau_{p} l_{e}}{g_{e}^{\prime}} .
$$

Computing $\sum_{h>2, \text { odd }} L_{m h, h}(h)$ results in the total harmonic coupling inductance between two phases, $L_{m h}$. This sum can be compared with the fundamental coupling under balanced excitation which is the magnetizing inductance $L_{m s}$

$$
L_{m h}=L_{m s}\left[\frac{1}{3 k_{1}^{2}} \sum_{h \text { odd }}^{\infty} \frac{k_{h}^{2}}{h^{2}}\right]=K_{H} L_{m s} .
$$

Note that under normal balanced three-phase wye connected operation, the triplen MMF harmonics cancel, resulting in no effective coupling for the triplen space harmonics. Nontriplen flux harmonics will still be present; however, their harmonic magnetizing inductances will be effectively shorted by the corresponding harmonic rotor circuit [10]. When the machine is saturated due to large flux levels, triplen flux harmonics will still be present. However, when the machine is not saturated, $L_{m h}$ typically can be ignored.

In (1), the slot and end-winding leakage fluxes have been included after rewriting (2) and (3) solely in terms of $i_{a s}$. This is possible since under normal operating conditions $i_{a s}=-i_{b s}-$ $i_{c s}$. For the purposes of the proposed test, this condition will not be present and therefore, the slot leakage and end-turn flux linkages must remain in the form of (2) and (3). In order to simplify notation, $L_{s l s}$ will be assumed to include both the slot self-leakage as well as the other nonslot leakage components such as the zigzag leakage inductance. Furthermore, $L_{m h}$ has also been neglected in (1).

Thus, (1) can be expanded to

$$
\begin{aligned}
\lambda_{a s}= & \left(L_{s l s}+L_{e w s}+2 L_{m h}+L_{m s}+L_{l k}\right) i_{a s} \\
& -\left(L_{s l m}+L_{e w m}+\frac{L_{m s}}{2}+L_{m h}\right) i_{b s} \\
& -\left(L_{s l m}+L_{e t m}+\frac{L_{m s}}{2}+L_{m h}\right) i_{c s} \\
& +L_{s r} i_{a r}-\frac{L_{s r}}{2} i_{b r}-\frac{L_{s r}}{2} i_{c r} .
\end{aligned}
$$

It should be remembered that the slot mutual inductance $L_{s l m}$, the end-winding mutual inductance $L_{\text {ewm }}$ and the harmonic coupling inductance $L_{m h}$ are different from the mutual inductance $L_{m s}$ since $L_{s l m}$ is strictly in the slot, $L_{\text {ewm }}$ is outside of 
the torque producing region of the machine and $L_{m h}$ crosses the air gap but is not involved in torque production while $L_{m s}$ crosses the air gap and is involved in torque production and so therefore it is the objective of this test.

\section{B. Induced Phase Voltage}

The voltage equation for the $a$-phase winding is

$$
v_{a s}=i_{a s} r_{s}+\frac{d \lambda_{a s}}{d t}
$$

If the $a$ - and $c$-phase windings are open circuited and only the $b$-phase winding is excited by utilizing the neutral connection, (11) becomes

$$
\begin{aligned}
v_{a s}=\frac{d}{d t}\left[-\left(L_{s l m}+L_{e w m}\right.\right. & \left.+\frac{L_{m s}}{2}+L_{m h}\right) i_{b s} \\
& \left.+L_{s r} i_{a r}-\frac{L_{s r}}{2}\left(i_{b r}+i_{c r}\right)\right] .
\end{aligned}
$$

Now, using the Fundamental Limit Theorem from Calculus, one can integrate both sides with respect to time from zero to infinity to obtain

$$
\begin{aligned}
\lambda_{a s}= & \int_{0}^{\infty} v_{a s} d t \\
= & -\frac{1}{2} \int_{L_{m s} i_{b s}(0)}^{L_{m s} i_{b s}(\infty)} d\left(L_{m s} i_{b s}\right)+\int_{L_{s r} i_{a r}(0)}^{L_{s r} i_{c r}(\infty)} d\left(L_{s r} i_{a r}\right) \\
& -\frac{1}{2} \int_{L_{s r} i_{a r}(\infty)}^{L_{i_{b r}}(0)} d\left(L_{s r} i_{b r}\right)-\frac{1}{2} \int_{L_{s r} i_{c r}(0)}^{L_{e w m} i_{b s}(\infty)} d\left(L_{s r} i_{c r}\right) \\
& \left.-\int_{L_{m h} i_{b s}(0)}^{L_{m h} i_{b s}(\infty)} d\left(L_{m h} i_{b s}\right)-\int_{s l m} i_{b s}\right) \\
& -\int_{L_{s l m} i_{i_{b s}}(0)}^{L_{L_{s w m}}(\infty)} d\left(L_{s l m} i_{b s}\right) .
\end{aligned}
$$

The machine is assumed to be initially not excited, so all of the machine currents are initially zero. If a voltage step function is applied to the $b$-phase winding, after the transients die out the rotor currents will return to zero. This fact simplifies (13) to the form

$$
\begin{aligned}
\lambda_{a s}= & -\int_{0}^{L_{s l m} i_{b s}(\infty)} d\left(L_{s l m} i_{b s}\right)-\int_{0}^{L_{e w m} i_{b s}(\infty)} d\left(L_{e w m} i_{b s}\right) \\
& -\int_{L_{m h} i_{b s}(0)}^{L_{m h} i_{b s}(\infty)} d\left(L_{m h} i_{b s}\right)-\frac{1}{2} \int_{0}^{L_{m s} i_{b s}(\infty)} d\left(L_{m s} i_{b s}\right) \\
= & -\left(L_{s l m}+L_{e w m}+L_{m h}+\frac{L_{m s}}{2}\right) i_{b s}(\infty) .
\end{aligned}
$$

For practical machine designs, the total leakage inductance $L_{l s}+L_{l r}$ is usually $20 \%$ or less of the magnetizing inductance (this would imply an in-rush current of approximately 5 p.u.). Typically, $L_{l s} \approx L_{l r}$ and so an upper estimate for $L_{l s} \approx 0.1 L_{m}$.
In a full-pitch machine the value of $L_{\text {slm }}$ and $L_{\text {ewm }}$ will be a maximum and it can be shown that $L_{s l m} \approx L_{s l s} / 3$ under these conditions [11]. Since $L_{l s}$ also includes other leakage terms in addition to $L_{s l s}$ and $L_{s l m}$, a conservative estimate for $L_{s l s}$ would be $L_{s l s} \approx L_{l s} / 2$. Next, it is evident that for practical machines $L_{s l m}>10 L_{\text {ewm }}$. Thus, $L_{s l m} \approx L_{m s} / 40$ and $L_{\text {ewm }} \approx L_{m s} / 400$

Equation (9) provides that $L_{m h}=K_{H} L_{m s}$. Assuming a full pitch, concentrated winding then $k_{h}=1$, for all $h$, resulting in $K_{H}=0.078$. Practical machines have distributed windings and are typically wound with a fractional pitch so that $k_{h}<1$ and so $K_{H}$ will be even smaller.

Thus, in (14), $L_{\text {slm }}$ and $L_{\text {ewm }}$ can be neglected with less than $2.75 \%$ error and $L_{m h}$ can be neglected with less than $7.8 \%$ error. Note that these errors cannot be strictly added because some of the underlying assumptions for each error calculation are contradictory. While the relative error due to the harmonics is larger than might be desired, $K_{H}$ can be calculated strictly based on the easily observed winding construction and then used to increase the accuracy of the measurement if so desired.

Under balanced three-phase excitation, the armature currents create a rotating magnetic flux in the air gap which is 1.5 times the magnitude of the flux due to one phase alone (6). Hence, on a per-phase basis, three-phase operation corresponds to the inductance $(3 / 2) L_{m s}$ [3]. In the proposed test, only one phase is excited and, therefore, (14) needs to be multiplied by 3 on both sides to obtain the conventional magnetizing inductance of the per-phase equivalent circuit.

These results imply that by measuring and then integrating the $a$-phase winding voltage when the b-phase winding is excited by the step function, the magnetizing inductance can be measured directly as

$$
L_{m}=\frac{3}{2} L_{m s}=-\frac{3 \lambda_{a s}}{i_{b s}} .
$$

Note that the result of this integration is entirely independent of the current induced variation of inductances $L_{m s}$ and $L_{s r}$ so that saturation is inherently included. This method determines the actual ratio of the flux linkage to the winding current and therefore correctly measures the chord or apparent inductance as opposed to the small-signal inductance [12].

\section{Theoretical Comparisons Between the DC and the $A C$ Measurements}

In order to compare, as a function of current, the value of the magnetizing inductance obtained by the de test to that obtained by the ac test it must be recognized that the dc current is equivalent to the peak of the ac current. Fig. 2 shows the same current level for ac and dc excitation. Hence, the dc current must be divided by $\sqrt{2}$ in order to be equivalent to the ac rms current. In the proposed dc test, only one phase is excited, while in the ac test, all three phases are excited. Therefore, the current of the dc test must be further divided by $3 / 2$ in order to obtain the same MMF as produced by a balanced three phase excitation. Thus, the graph which represents the magnetizing inductance as a function of the dc current has to be shifted by dividing its current axis by $(3 / 2) \sqrt{2}$ in order to calibrate to balanced average phase current. 


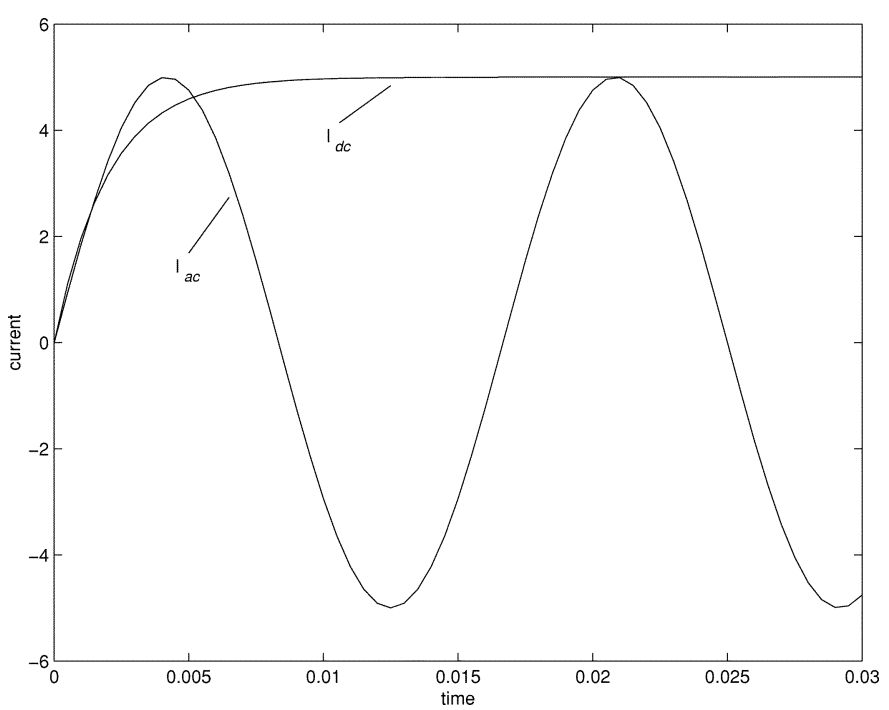

Fig. 2. Comparison between tests with ac and dc excitation.

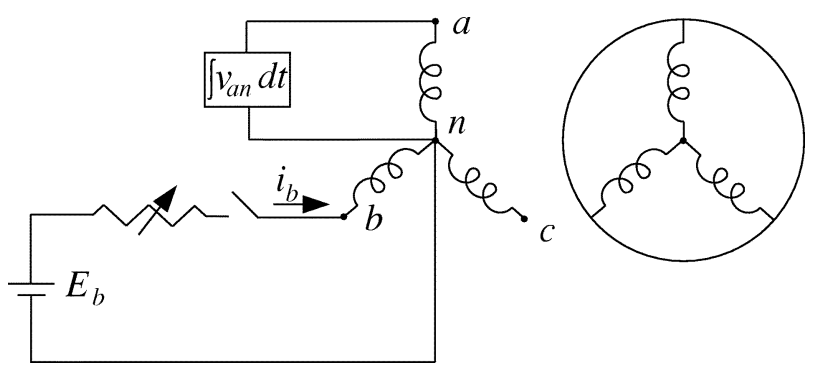

Fig. 3. Experimental setup.
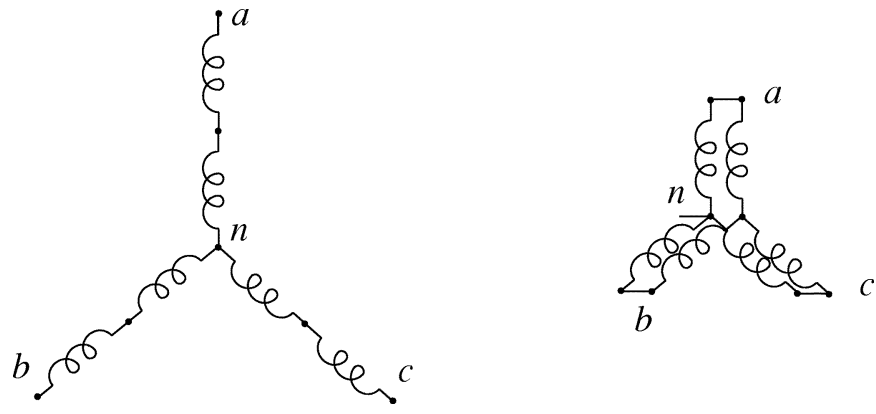

Fig. 4. Dual-voltage machine connections.

\section{Proposed Experimental Method}

The experimental setup is shown in Fig. 3.

The series resistor limits the applied current to the desired value. When the switch is closed, the measured voltage is integrated to obtain the flux linkage $\lambda$. The steady-state current $i$ is measured and (15) is used to compute $L_{m}$. This measurement can be performed with different current levels to obtain a magnetizing inductance versus current curve.

This test can be conducted with a step increase (turn-on) or step decrease (turn-off) in voltage. However, experiments indicate that the turn-on method is less noisy because the energy stored in the stator leakage inductance creates an arc across the switch which affects the calculation during turn-off. Finally, it should be noted that measurements at lower current levels may be challenging because the induced voltages will be smaller and

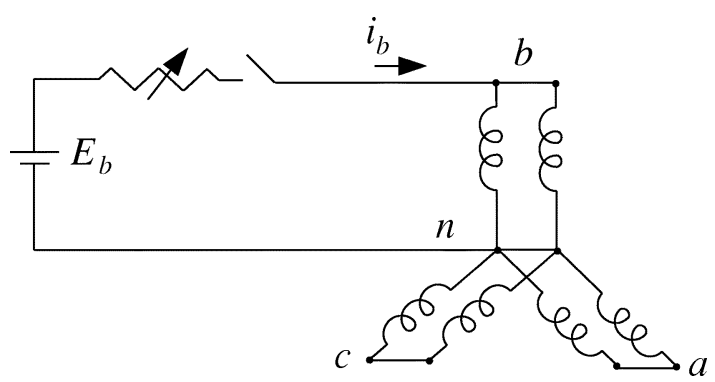

Fig. 5. Low-voltage machine connection for $L_{m}$ measurement.

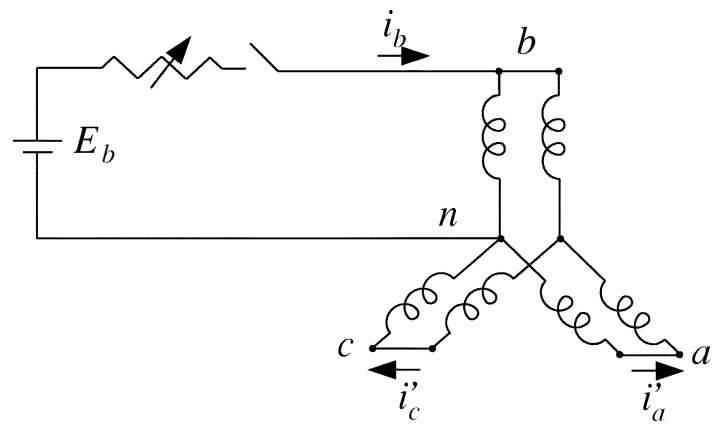

Fig. 6. Machine connection with circulating currents.

hence it more difficult to obtain accurate results. With careful attention to proper measurement techniques and the averaging of repeated measurements, reasonable results are still possible.

If the machine is a dual-voltage type, then the windings can be connected either in series or in parallel as in Fig. 4. In both cases, the neutral has to be made accessible in order to perform the measurements. If the first and second sets of windings are connected in parallel as in Fig. 5 during normal operation, both neutrals need to be shorted in order to apply the proposed method. If both neutrals are not connected as in Fig. 6 then the unbalanced currents $i_{a}^{\prime}$ and $i_{c}^{\prime}$ flow in the two three-phase groups and this can cause measurement errors.

\section{EXPERIMENTAL RESULTS}

In order to verify the results obtained by the proposed method, the traditional no-load and locked-rotor tests were also performed. From the per-phase equivalent circuit, the sum of the magnetizing and the stator leakage inductance was obtained. This measurement was performed for a number of different flux levels for two different sized machines.

The proposed method was first tested on a dual-voltage 1-hp 230/115-V 86-Hz motor. The windings were connected in series and access was provided to the neutral point. The magnetizing inductance $L_{m}$ as a function of dc current obtained from the dc test is shown in Fig. 7 while the sum of the magnetizing inductance and the stator leakage $L_{m}+L_{l s}$ as a function of the rms current obtained in the no-load test (IEEE Standard 112-1996 [4]) is shown in Fig. 8.

In order to compare these two figures, the dc test curve was shifted by $(3 / 2) \sqrt{2}$ along the current axis and plotted on the same graph with the results from the no-load test. This comparison is shown in Fig. 9. The difference between the two curves clearly represents the stator leakage inductance $L_{l s}$. 


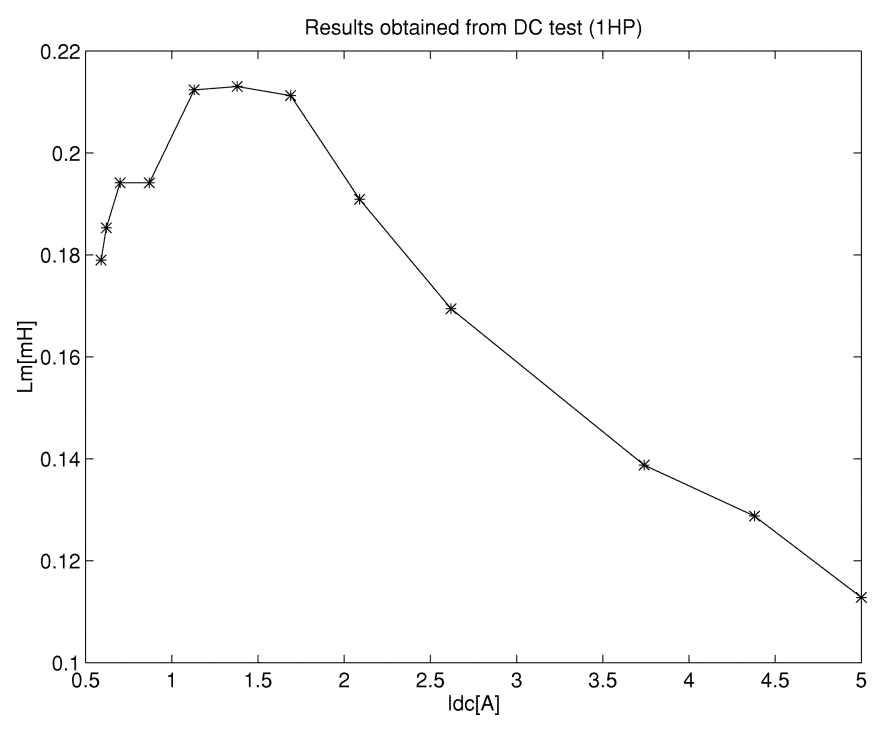

Fig. 7. 1-hp dc excitation test $L_{m}$.

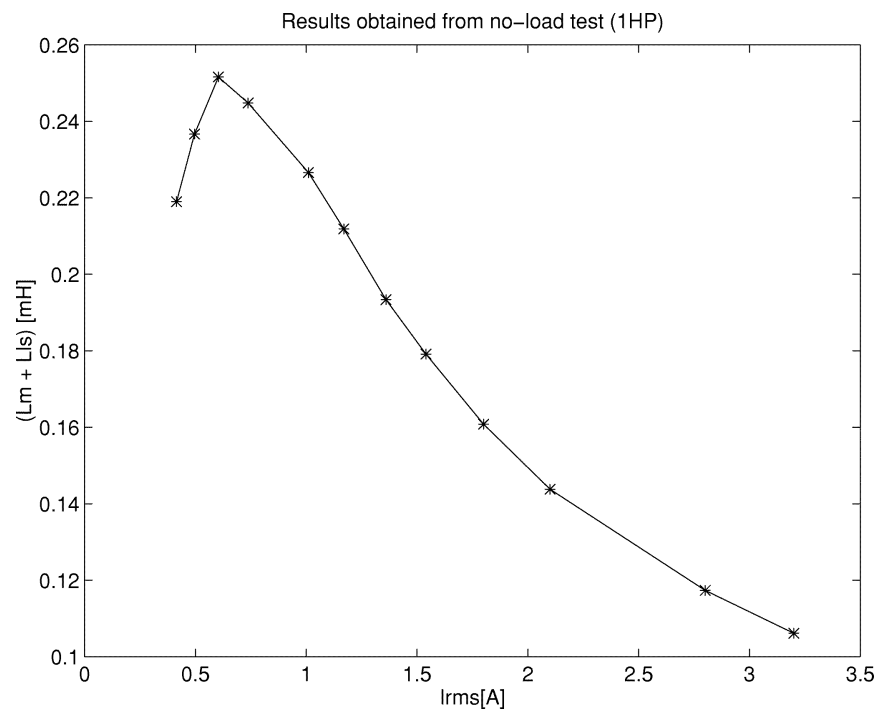

Fig. 8. 1-hp no-load test $L_{m}+L_{l s}$.

Next, the proposed method was tested on a dual-voltage 15-hp 460/230-V 60-Hz motor. When the switch is closed as shown in Fig. 3, voltage $\left(V_{a n}\right)$ must be measured and integrated. The measured voltage $V_{a n}$, its integrated value (flux) and the dc current $i_{b}$ are shown in Fig. 10. This oscilloscope trace can be used to calculate one point on the $L_{m}$ verses $I_{d c}$ curve. The current levels were varied over a wide range and the measurement repeated to obtain the complete curve.

In order to verify the value of the magnetizing inductance obtained by the proposed test, the manufacturer's proprietary design program was used to obtain a theoretical $L_{m}$ versus $I$ curve. This theoretical curve was compared with the empirical curves obtained by using the proposed and traditional method using the locked-rotor and no-load tests of IEEE Standard 112-1996 [4]. The total leakage inductance as measured by the locked-rotor test is shown in Fig. 11. The test was performed for two different phase windings. The effect of saturation is evident by the decrease in the leakage inductance. Note that above 8-10 A, the inductance is approaching a constant value. Finally, an eighth

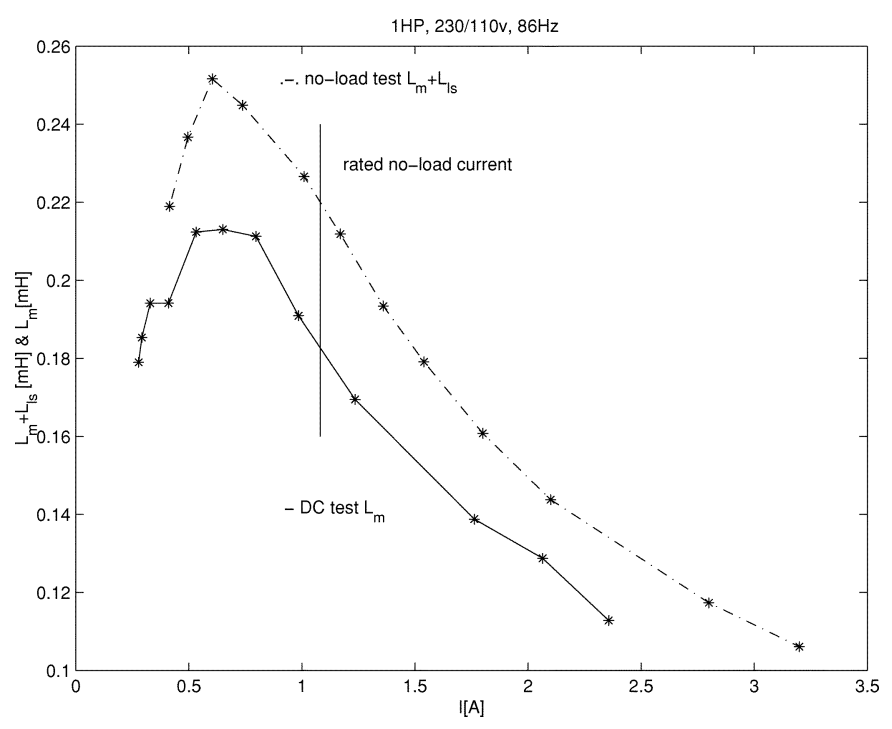

Fig. 9. 1-hp dc excitation $L_{m}$ and no-load test $L_{m}+L_{l s}$.

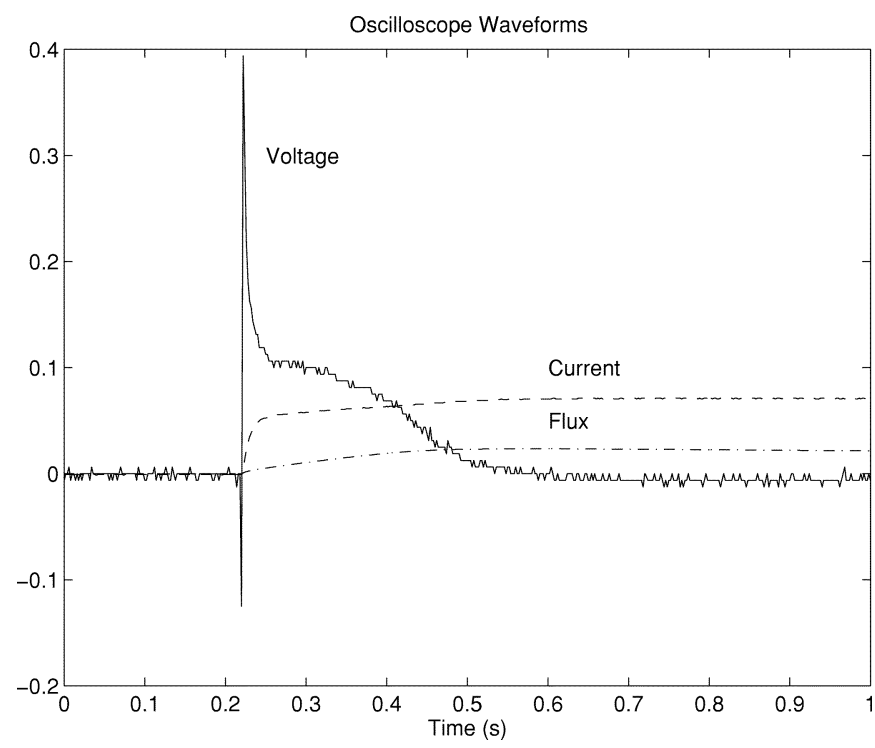

Fig. 10. 15-hp oscilloscope traces for $V_{a n}, \int V_{a n} d t$, and $i_{b}$.

order polynomial was curve fit to the data points and this expression will be used to compare the proposed test to the traditional no-load test.

The comparison between the proposed method, traditional method, theoretical and proposed method plus measured leakage is shown in Fig. 12. Note the expanded inductance scale. The traditional no-load measurement was performed on two different phase windings and in the figure, the results are indicated by an asterisk and a plus for phases $A$ and $B$, respectively. In this figure, the theoretical $L_{m}$ is shown as a solid line and the proposed method shows good agreement with the theoretically predicted $L_{m}$. The difference between the proposed method and the traditional method is approximately constant for currents above 8-10 A, which is where the leakage inductance is approximately constant. To further validate the results, the curve-fit expression for the leakage inductance was used to compute a value of $L_{l s}+L_{l r}$ for each current used in the proposed method. Assuming that $L_{l s}: L_{l r}=60: 40$, 


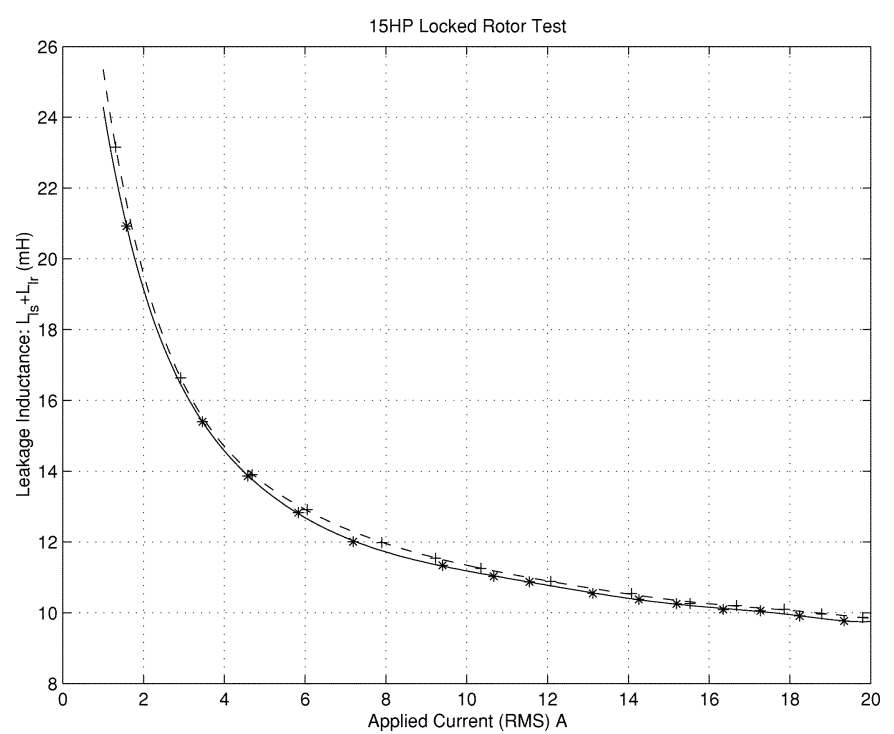

Fig. 11. 15-hp experimental leakage inductance.

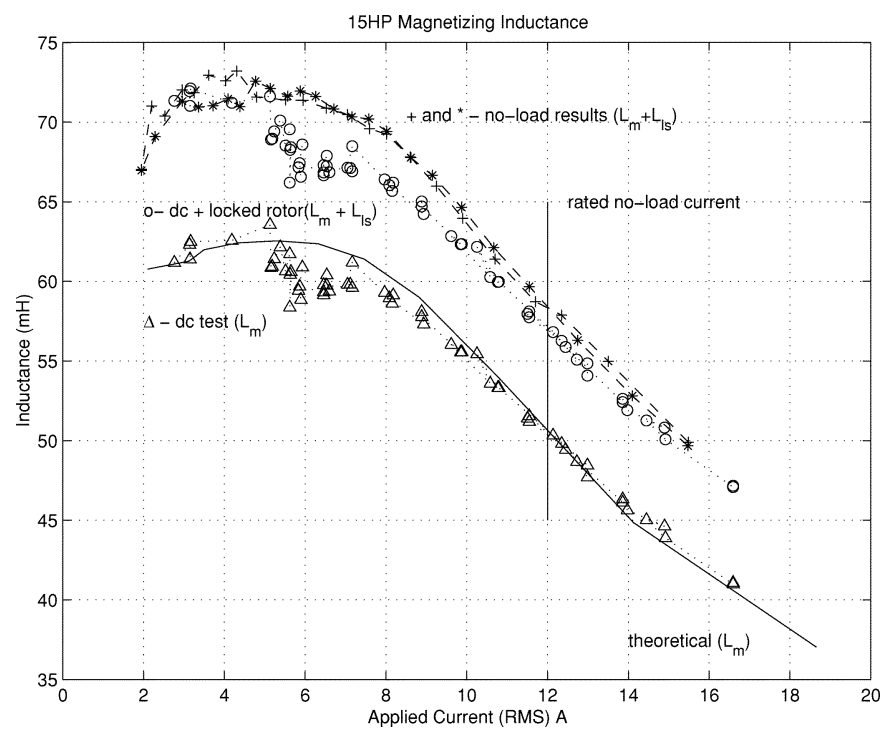

Fig. 12. 15-hp theoretical and experimental $L_{m}$.

the estimate of $L_{l s}$ was added to the values of $L_{m}$ from the proposed test. This resulting sum matches very closely to the results of the traditional no-load test.

The vertical line indicates the rated no-load current. Its intersection with the inductance curves shows the location of the magnetizing inductance $L_{m}$ at rated no-load current for each method. From these curves, it is clear that the proposed method calculates the magnetizing inductance accurately.

Having identified the magnetizing inductance, it is evident that the stator leakage inductance can now be uniquely determined from the no-load test. The difference between the traditional no-load and proposed dc test results in Fig. 12 represents the stator leakage of the 15-hp motor. In this case, the stator leakage inductance is approximately $7 \mathrm{mH}$.

\section{CONCLUSION}

This paper has presented a new approach to identify the induction machine magnetizing inductance by using a dc excitation test with the motor at standstill. Hence, for vector drive applications which require knowing the magnetizing inductance for the purpose of control, this needed parameter can be measured in situ using this test. The proposed method measures only the magnetizing inductance and makes it possible to calculate stator leakage inductances separately without any approximation or iterative methods. Specifically, the difference between the two curves in Fig. 9 or the no-load and dc test curves in Fig. 12 directly represents the stator leakage inductance. Being essentially a pulsed dc measurement, the iron losses incurred during the measurement are small and, therefore, they minimally affect the measurement. Verification of the proposed method was performed by comparing the inductance measured by this method with the theoretical value of the inductance calculated from a manufacturer's proprietary design program. Test results clearly show an improvement as compared to the traditional no-load and locked-rotor test. Verification of the proposed method was also performed by comparing the magnetizing inductance measured by this method with the total inductance (the sum of the magnetizing and the stator leakage inductance) calculated by a no-load test over a range of current. A constant difference between these two inductances was observed between both methods as the current is increased. Since the difference between the two inductance values corresponds to the stator leakage inductance, this result indicates that the proposed method correctly measures the effects of saturation.

\section{ACKNOWLEDGMENT}

The authors thank D. Hyppio, Marathon Electric; D. Novotny, University of Wisconsin, Madison; D. Saban, formerly with the General Electric Motors Division; and R. King and G. Kliman, General Electric Global Research Center, for their valuable comments.

\section{REFERENCES}

[1] P. L. Alger, Induction Machines: Their Behavior and Uses, 2nd ed. New York: Gordon and Breach, 1970.

[2] M. G. Say, The Performance and Design of Alternating Current Machines, 3rd ed. London, U.K.: Sir Isaac Pitman, 1958.

[3] A. E. Fitzgerald, C. Kingsley Jr., and S. D. Umans, Electric Machinery. New York: McGraw-Hill, 1952.

[4] IEEE Standard Test Procedure for Polyphase Induction Motors and Generators, IEEE Std. 112-1996, 1996.

[5] S. I. Moon and A. Keyhani, "Estimation of induction machine parameters from standstill time-domain data," IEEE Trans. Ind. Applicat., vol. 30, pp. 1609-1615, Nov./Dec. 1994.

[6] J.-K. Seok, S.-I. Moon, and S.-K. Sul, "Induction machine parameter identification using PWM inverter at standstill," IEEE Trans. Energy Conversion, vol. 12, pp. 127-132, June 1997.

[7] J. R. Willis, G. J. Brock, and J. S. Edmonds, "Derivation of induction motor models from standstill frequency response tests," IEEE Trans. Energy Conversion, vol. 4, pp. 608-613, Dec. 1989.

[8] C. V. Jones, The Unified Theory of Electrical Machines. London, U.K.: Butterworth, 1967.

[9] D. W. Novotny and T. A. Lipo, Vector Control and Dynamics of Induction Motor Drives. Oxford, U.K.: Clarendon, 1996.

[10] P. L. Alger, The Nature of Polyphase Induction Machines. New York: Wiley, 1951.

[11] T. A. Lipo, Introduction to AC Machine Design. Madison, WI: Wisconsin Power Electronics Research Center-Univ. of Wisconsin Press, 1996, vol. 1. 
[12] J. Melkebeek and D. W. Novotny, "The influence of saturation on induction machine drive dynamics," IEEE Trans. Ind. Applicat., vol. IA-19, pp. 671-681, Sept./Oct. 1983. 